\title{
Erratum to: Mössbauer analysis of coal coke samples from Samacá, Boyacá, Colombia
}

\author{
W. A. Pacheco Serrano • D. Quintão Lima • \\ J. D. Fabris
}

Published online: 24 July 2013

(C) Springer Science+Business Media Dordrecht 2013

\section{Erratum to: Hyperfine Interact \\ DOI 10.1007/s10751-013-0873-y}

This article was published with an erroneous version of Table 1. Please find the correct Table 1 on this page that should be regarded by the reader as the final version.

Table 1 Hyperfine parameters measured by ${ }^{57} \mathrm{Fe}$ Mössbauer spectroscopy at room temperature for coal coke samples after chemical treatment with $\mathrm{NaOH} 5 \mathrm{~mol} \mathrm{~L}{ }^{-1}$

\begin{tabular}{lllllll}
\hline Sample & $\delta / \mathrm{mm} \mathrm{s}^{-1}$ & $\varepsilon / \mathrm{mm} \mathrm{s}^{-1}$ & $\Delta / \mathrm{mm} \mathrm{s}^{-1}$ & $\mathrm{~B}_{\mathrm{hf}} / \mathrm{T}$ & $\mathrm{RA} / \%$ & Phase \\
\hline G01 & $0.371(2)$ & $-0.208(3)$ & & $51.57(1)$ & $76(1)$ & $\mathrm{Hm}$ \\
& $0.28(2)$ & & $0.92(3)$ & & $24(1)$ & $\mathrm{Fe}^{3+}$ \\
L02 & $0.368(1)$ & $-0.237(2)$ & & $51.349(7)$ & $60(1)$ & $\mathrm{Hm}$ \\
& $0.283(3)$ & & $0.867(4)$ & & $40(1)$ & $\mathrm{Fe}^{3+}$ \\
M03 & $0.369(2)$ & $-0.216(3)$ & & $50.98(1)$ & $63(1)$ & $\mathrm{Hm}$ \\
& $0.306(4)$ & & $0.847(7)$ & & $37(1)$ & $\mathrm{Fe}^{3+}$ \\
\hline
\end{tabular}

$\delta=$ isomer shift relative to $\alpha \mathrm{Fe} ; \Delta=$ quadrupole splitting, $\varepsilon=$ quadrupole shift; $\mathrm{B}_{\mathrm{hf}}=$ hyperfine magnetic field; $\mathrm{RA} / \%=$ relative subspectral area; $\mathrm{Hm}=$ hematite; $\mathrm{Fe}^{3+}=$ (super) paramagnetic high spin ferric iron oxide(s). Numbers in parentheses are uncertainties over the last significant digit corresponding to the value, as outputted by the least squares-fitting computer program

The online version of the original article can be found under http://dx.doi.org/10.1007/s10751-013-0873-y.

W. A. Pacheco Serrano $(\varangle)$

Escuela de Física, Universidad Pedagógica y Tecnológica de Colombia, UPTC,

Tunja, Boyacá, Colombia

e-mail: william.pacheco@uptc.edu.co

D. Quintão Lima · J. D. Fabris

Departamento de Química, ICEx, Universidade Federal de Minas Gerais,

Belo Horizonte, MG, Brasil

D. Quintão Lima

e-mail: dianaquintao@yahoo.com.br

J. D. Fabris

Universidade Federal dos Vales do Jequitinhonha e Mucuri (UFVJM), 39100-000 Diamantina, MG, Brasil

e-mail: jdfabris@ufmg.br 